\title{
Terapia de rehabilitación cognitiva, calidad de vida de pacientes con enfermedad de Parkinson y cuidadores
}

\author{
DOI 10.5377/alerta.v4i3.10291
}

Aída Camila Suárez Jiménez ${ }^{1^{*}}$, Melissa Esmeralda Moreno Ramírez ${ }^{2}$, Susana María Montoya Romero ${ }^{3}$

1, 2 y 3. Universidad Dr. José Matías Delgado, Santa Tecla, La Libertad, El Salvador.

${ }^{*}$ Correspondencia

$\square$ cams167j@hotmail.com

1. (1) $0000-0003-2038-2790$

\section{Resumen}

La enfermedad de Parkinson es la segunda patología neurodegenerativa más recurrente, progresiva y crónica que perjudica a pacientes y cuidadores. Es fundamental describir la calidad de vida en pacientes y cuidadores posterior a la terapia de rehabilitación cognitiva. Por ello se elaboró un artículo de revisión mediante una búsqueda en bases de datos, PubMed, Google Scholar, SciELO, empleando artículos originales, ensayos clínicos, de revisión, entre otros, publicados en los últimos cinco años en español e inglés. El deterioro cognitivo en los pacientes de forma prematura conlleva a mayor riesgo de demencia, que se manifiesta con repercusión en las actividades de la vida diaria, como en la calidad de vida pacientecuidador. Mientras más avanzado se encuentre el estadio de la enfermedad, los pacientes con deterioro cognitivo generan mayor dependencia de sus cuidadores, empeorando la calidad de vida de ambos. La terapia de rehabilitación cognitiva pretende mejorar la calidad de vida de pacientes con enfermedad de Parkinson y sus cuidadores; actualmente se describen terapias de rehabilitación cognitiva basada en ejercicio físico. Al fortalecer la cognición mediante terapias de rehabilitación basadas en actividad física, el paciente presenta mayor independencia, lo cual disminuye la carga del cuidador, generando mayor empatía entre ambos.

Palabras clave

Enfermedad de Parkinson, cognición, rehabilitación, cuidadores, ejercicio físico.

\begin{abstract}
Parkinson's disease is the second most common, progressive and chronic neurodegenerative pathology that affects patients and caregivers. It is imperative to describe the quality of life in patients and caregivers after receiving cognitive rehabilitation therapy. A review article was elaborated through a database search, PubMed, Google Scholar, SciELO, using original articles, clinical trials, review articles, among others, published in the last five years in both Spanish and English. Cognitive impairment in patients with Parkinson's disease prematurely leads to a higher risk of dementia; it alters daily activities as well as patient-caregiver life. At a more advanced stage of the disease, the patient requires additional support, worsening their welfare. Rehabilitation therapy intends to improve the quality of life in patients with Parkinson's disease and their caretakers. Cognitive rehabilitation therapies based on physical exercise are currently described. As we improve cognition through physical activities, the subject exhibits more independence to work on their daily duties, which at the same time diminishes the caregiver overload, strengthening the empathy between them.
\end{abstract}

Keywords

Parkinson's disease, cognition, rehabilitation, caregivers, exercise.

\section{Introducción}

La enfermedad de Parkinson (EP) es la segunda patología neurodegenerativa más recurrente, progresiva y crónica que perjudica a pacientes y cuidadores. Ocurre en más del $1 \%$ de personas mayores de 60 años, con predominio del sexo masculino'. Es una patología compleja que requiere un abordaje multidisciplinario para enlentecer el deterioro cognitivo (DC) y mejorar la calidad de vida². f ACCESO ABIERTO

Cognitive rehabilitation therapy, quality of life in Parkinson's disease patients and their caregivers

\section{Citación recomendada:} Suarez Jiménez AC, Moreno Ramírez ME, Montoya Romero SM. Terapia de rehabilitación cognitiva, calidad de vida de pacientes con enfermedad de Parkinson y cuidadores. Alerta. 2021;4(3):143-150. DOI: 10.5377/alerta.v4i3.10291

Recibido:

15 de octubre de 2020

Aceptado:

9 de junio de 2021

Publicado:

26 de julio de 202

Contribución de autoría: ACSJ', MEMR ${ }^{2}$, SMMR $^{3}$; búsqueda bibliográfica, redacción y edición del manuscrito.

Conflicto de intereses: Autores declaran no tener conflicto de interés. 
La prevalencia es mayor en Europa y América del Norte, donde se reportan $20 \mathrm{ca}-$ sos por cada 100000 habitantes (media de 57 años)33; aproximadamente, 10 millones de personas en todo el mundo sufren de EP. La causa no está bien definida actualmente, no obstante, pueden presentarse factores extrínsecos e intrínsecos; además, los factores genéticos se han asociado en el $10 \%{ }^{4-5}$.

Existen diversos síntomas a lo largo de la enfermedad, clasificados en motores y no motores, predominantemente el DC, que se define por lentitud de los procesos mentales, falta de iniciativa para ejecutar tareas, apatía, disminución de atención y, ocasionalmente, depresión ${ }^{6}$.

Los pacientes con mayor DC requieren más horas de atención, generando dependencia de sus familiares, quienes desatienden actividades diarias; el deterioro de las condiciones de vida de pacientes con EP es un determinante que tiene resultados negativos en los cuidadores, lo cual significa que al mejorar el DC se incrementa la calidad de vida de ambos?

\section{Metodología}

Se elaboró un artículo de revisión narrativa por medio de búsqueda en las bases de datos PubMed, Google Scholar y SciELO. Se consultaron artículos originales, estudios randomizados, ensayos clínicos y de revisión, en español y en inglés, publicados en los últimos 5 años (2015 a 2020) y se excluyó la literatura gris. Se aplicaron los siguientes conectores booleanos: enfermedad de Parkinson AND calidad de vida, terapia de rehabilitación cognitiva AND terapia farmacológica, Parkinson's disease AND Tai Chi, quality of life AND caregivers.

Por lo descrito anteriormente, con el fin de disminuir el impacto socioeconómico y aumentar la empatía de ambas partes, se pretende describir la calidad de vida en pacientes con EP y sus cuidadores al recibir terapia de rehabilitación cognitiva basada en ejercicio físico.

\section{Desarrollo}

\section{Generalidades}

La EP es la disfunción de la vía dopaminérgica de la sustancia negra por alteración en la expresión de proteínas o mal plegamiento, con el hallazgo patognomónico llamado cuerpos de Lewy, que son fibrillas similares a amiloides, seguido de alteración de estructura y función neuronal por medio de degeneración temprana, irreversible y progresiva ${ }^{8}$. La disminución de la función mito- condrial y la desregulación de homeostasis intracelular del calcio producen muerte en neuronas dopaminérgicas posterior la inhibición del núcleo subtalámico, limitando la actividad locomotora?

Los síntomas clínicos se clasifican en motores y no motores. Entre los motores, el principal es la bradicinesia, manifestada por el inicio lento de movimientos voluntarios y la reducción progresiva de la velocidad de estos, acompañado de rigidez muscular, tremor e inestabilidad postural. Entre los no motores están los trastornos del sueño, como insomnio, pesadillas, anhedonia, depresión y ansiedad. El diagnóstico clínico se basa en tres pasos: primero, la bradicinesia; segundo, la exclusión de otras causas de parkinsonismo, y tercero, al menos tres de los criterios clínicos del banco de cerebros de la sociedad de enfermedad de Parkinson del Reino Unido ${ }^{10}$.

Bolaños y col. clasifican la EP, según su estudio en el Hospital Central Militar basados en la escala de Hoehn and Yahr (Tabla 1), que valora progresión y gravedad de la enfermedad en estadios del 1 al $5^{11}$.

Tabla 1. Escala de Hoehn and Yahr que valora progresión y gravedad de la enfermedad de Parkinson

\begin{tabular}{cl}
\hline Estadio & Descripción \\
\hline 0 & Sin signo de enfermedad \\
1 & Enfermedad unilateral \\
1,5 & Unilateral más compromiso axial \\
2 & Bilateral, sin compromiso del equilibrio \\
2,5 & Bilateral leve, con recuperación en la \\
& prueba de empuje \\
3 & $\quad$ De leve a moderado, bilateral, alguna \\
4 & inestabilidad \\
& Grave inestabilidad, capacidad para \\
5 & Silla de ruedas o postrado en la cama
\end{tabular}

Fuente: Bolaños M, Meneses F, Samano J, Hernández C, Torres C. Estimulación cerebral profunda en núcleo subtalámico bilateral en pacientes con enfermedad de Parkinson en el Hospital Central Militar. Rev Sanid Milit Mex. 2019;73(2), 90-95. Disponible en: https:// revistasanidadmilitar.org/index.php/rsm/article/ view/16

\section{Deterioro cognitivo}

Anteriormente se afirmaba que el DC era exclusivo de estadios avanzados de EP; no obstante, se ha determinado que puede manifestarse en etapas primarias y los síntomas iniciales pueden ser inadvertidos clínicamente ${ }^{12}$. Biundo y col. agregaron que al iniciar de manera prematura conllevaba a un mayor riesgo de desarrollar demencia, provocando inestabilidad y más ingresos 
hospitalarios y requiriendo intervenciones tempranas ${ }^{13}$.

Asimismo, Pico y col. establecen que el diagnóstico en etapas iniciales es difícil, ya que los síntomas clínicos no son concluyentes. También determinaron que la demencia puede presentarse incluso años posteriores al diagnóstico ${ }^{14}$. Debido a la importancia de la detección del DC, Piggot y col., en su estudio de cognición normal en la EP, acordaron que la puntuación inicial de la escala de Hoehn \& Yahr era un factor predictor para el desarrollo de la enfermedad ${ }^{15}$.

En efecto, el DC genera discapacidad funcional con prevalencia del 20-50\%, siendo la disfunción ejecutiva uno de los componentes más afectados, como la disminución de la capacidad resolutiva, la planeación y la memoria de trabajo. El tiempo de evolución puede variar en cada individuo; sin embargo, tarda aproximadamente 10 años alcanzar un estadio mayor al tres en la mayoría de afectados y en raras ocasiones menos de cinco años ${ }^{16}$. Mayorga y col. establecieron en el año 2018 que podía ser afectada la atención, el aprendizaje, el lenguaje, el reconocimiento social y la pérdida parcial o total de habilidades obtenidas anteriormente ${ }^{17}$.

En su trabajo realizado en la Universidad Autónoma de Barcelona, Bobadilla determinó que al inicio de la enfermedad los síntomas cognitivos pueden ser definidos por molestias no relacionadas por parte del paciente, en su mayoría causados por fallo de inervación dopaminérgica, demostrando la importancia del estudio neuropsicológico en etapas tempranas como factor predictivo de demencia ${ }^{18}$.

Smith y col., en su estudio de 53 casos de sujetos con EP y 23 controles sobre afecciones cognitivas tempranas, específicamente el lenguaje, determinaron que pacientes enfermos presentaron más pausas al elaborar frases, articulaban menos palabras por minuto y las oraciones formuladas adecuadamente eran escasas por su función cognitiva alterada ${ }^{19}$.

En una investigación realizada en el Hospital de Stavanger se encontró en $62 \%$ de pacientes con EP diagnosticados con DC que habían manifestado demencia en cuatro años y de los que no presentaban, el 20\% lo hizo posteriormente; es decir, gran porcentaje de los sujetos progresaba en corto y mediano plazo a demencia, acompañada de síntomas depresivos ${ }^{20}$.

El DC y los síntomas asociados no solo perjudican al sujeto sino también a su encargado, desarrollándose el fenómeno de carga del cuidador. Genç y col., en 2019, lo definieron como la tensión que experimenta el principal miembro de la familia delegado ante la enfermedad crónica, la discapacidad, la vejez y en EP a los síntomas neuropsiquiátricos, los efectos adversos de medicamentos, la progresión de la enfermedad $^{21}$. Además, Grün y col. manifestaron que el estado de salud de los cuidadores se ve afectado en un $40 \%$ y que la tasa de depresión es superior a la población general, alterando su calidad de vida 22 .

\section{Calidad de vida en enfermos}

La Organización Mundial de la Salud (OMS) define calidad de vida como la percepción del sujeto sobre su postura en la vida, en el entorno de cultura y valores en los cuales vive, congruentes con sus fines, expectativas, estándares y preocupaciones ${ }^{23}$.

Además, consta de un componente subjetivo y un objetivo. El primero se basa en la satisfacción de vida que tiene el paciente y el segundo va encaminado hacia factores sociales, físicos y psíquicos del mismo; conociendo los componentes, se aplica la asociación de cuáles son afectados en el DC en la EP24.

Los pacientes con EP, según Aponte y col., manifiestan más alteraciones de la calidad de vida, de acuerdo con el estadio de la enfermedad que presentan en relación a los cambios en la función física y el estado emocional, aumentando la morbilidad y el ingreso hospitalario ${ }^{25}$. Los factores asociados con el entorno, la salud y el bienestar pueden analizarse por medio de escalas como PDQ-3926.

El PDQ-39 es una prueba que tiene como objetivo la valoración física, emocional y psicosocial de la calidad de vida de pacientes con EP; consta de 39 ítems con ocho apartados distribuidos según movilidad del paciente, actividades diarias, bienestar emocional, estigma, soporte social, estado cognitivo, comunicación y malestar con su cuerpo, realizando un interrogatorio para indagar la frecuencia de dificultades en el último mes ${ }^{27}$.

Aunado a ello, Ellingson y col., en un estudio realizado en Estados Unidos con 45 sujetos con EP, determinaron que el estilo de vida sedentario se asociaba a mayor puntaje en PDQ-39 y en subescalas de morbilidad, DC y problemas de comunicación; igualmente, se estableció que había un decremento más significativo en la calidad de vida en personas que utilizaban su tiempo viendo televisión, por lo tanto, el ejercicio físico es beneficioso a pesar de la difícil adherencia a un cambio de vida saludable ${ }^{28}$.

Takahashi y col., de la Universidad Kitasato en Japón, comprobaron una relación estadísticamente significativa de PDQ-39 
respecto a escalas de valoración de función motora y de actividades de la vida diaria. Se utilizó la prueba SEIQoL-DW para valorar el dominio de la calidad de vida más importante para los pacientes, relaciones sociales, principalmente con familia y amigos. Al mismo tiempo, la comunicación también es evaluada en PDQ-39, evidenciando que los pacientes con problemas para hablar o entablar una conversación adecuada suelen sentirse ignorados, creando sentimientos inseguridad y disminución del bienestar ${ }^{29}$.

Lawson y col., en un estudio de cohorte longitudinal realizado en un periodo 36 meses con 158 participantes clasificados, empleando el Mini Mental Test en estado cognitivo normal y DC medio, demostraron incremento de 9 puntos en el test PDQ-39 en pacientes con DC medio, predominantemente por depleción de la atención, memoria ejecutiva y actividades de la vida diaria, generando menor bienestar. Conjuntamente, se determinó peor calidad de vida en pacientes con demencia ${ }^{30}$.

\section{Calidad de vida de los cuidadores}

Papaterra, en una investigación ejecutada en 2017, establece que debe ser definido el estadio de la EP por su relación en la calidad de vida y la de sus cuidadores ${ }^{31}$, debido a que, según el grado de progresión, el cuidador realiza mayor esfuerzo, impactando negativamente en aspectos sociales y económicos y, por ende, alterando su calidad de vida e indirectamente la del enfermo ${ }^{32}$.

Asimismo, por ser una enfermedad neurodegenerativa requiere de cuidados por largos períodos, siendo el principal soporte un miembro del núcleo familiar. Anaut y col. caracterizaron al cuidador principal en su mayoría como mujer, casada, promedio de 50 años, hija del paciente, sin remuneración y jubilada, dificultando la sostenibilidad económica ${ }^{33}$.

Por lo antes mencionado, la calidad de vida en cuidadores de pacientes con EP presenta mayor relación de sobrecargaansiedad al ser alterados ejes económicos, emocionales y sociales ${ }^{34}$, siendo necesaria la intervención psicoterapéutica para mejorar las alteraciones en la calidad de vida de ambas partes ${ }^{35}$. Genç y col., en su estudio mencionado con anterioridad demostraron que un $40-50 \%$ del binomio presentaban síntomas depresivos asociados a síntomas no motores y eran incrementados por la fatiga, acrecentando a su vez la dependencia.

En Singapur, Tan y col. mostraron en el 2019 la asociación entre carga del cuidador y pacientes en estadios avanzados en conjunto con la discapacidad grave, requiriendo en promedio de ocho horas diarias, seis años de cuido; además, en un cuarto de sus participantes lograron verificar la importancia de la realización de grupos de apoyo y educación, mejorando la calidad de vida en el binomio ${ }^{36}$.

La sobrecarga es el principal factor que afecta a los cuidadores, por lo cual García y col., en su estudio, abordaron al encargado principal a través de la escala de Zarit, que valora la sobrecarga en tres componentes: impacto del cuidado, relación con el paciente con EP y percepción de la eficacia de su desempeño para evaluar el progreso, manifestaron que al reducir la sobrecarga fortalecían la empatía y aumentaba el bienestar de $\operatorname{ambos}^{37}$.

Rodríguez-Violante y col. refieren que, en estadios tardíos, los pacientes tienen mayor dificultad para realizar actividades cotidianas, y por ende, dependen más del cuidador. Pese a ello, la edad juega un rol importante; mientras más joven sea el cuidador, manifiesta mayor carga. Otras condiciones que generan preocupación en los cuidadores son el aspecto económico, la gravedad y el tiempo de la patología, los síntomas motores y no motores (como la depresión), las posibles caídas, entre otras, que alteran la calidad de vida de cuidador y paciente. Por lo tanto, mientras más satisfactorio sea el bienestar del paciente, la labor del cuidador disminuye ${ }^{38}$.

\section{Terapia de rehabilitación cognitiva en pacientes y cuidadores}

Las terapias farmacológicas y no farmacológicas en conjunto pretenden mejorar la calidad de vida de los pacientes con EP apoyando la movilidad, disminuyendo molestias por mala postura y aliviando la respiración. La terapia cognitiva en estadios tempranos puede prevenir o retrasar la distorsión de la movilidad y las capacidades funcionales, proporcionando mayor independencia al paciente y disminuyendo la carga del cuidador ${ }^{39}$.

La levodopa ha sido el medicamento de elección para fortalecer la calidad de vida en EP40; aunado a ello, las medidas no farmacológicas coadyuvan enlenteciendo el progreso de la enfermedad y disminuyendo algunas complicaciones a través de educación, la alimentación adecuada y la terapia de rehabilitación cognitiva, con el objetivo de restablecer el aspecto físico y mental para brindar mayor autonomía al paciente ${ }^{41}$.

La terapia de rehabilitación cognitiva radica en un conjunto de procedimientos, como ejercicio físico, memoria, atención, lenguaje, ejecución, planificación, fisioterapia, además de terapia ocupacional; sin 
embargo, el enfoque de esta revisión bibliográfica se basará en el componente de la actividad física. La Universidad Nacional Federico Villarreal, de Perú, llevó a cabo en 2019 un estudio donde se aplicó la rehabilitación cognitiva por medio de ejercicios en el hogar sobre visualización, asociación y repetición que fortalecieron las funciones ejecutivas como flexibilidad cognitiva, memoria semántica y planificación ${ }^{42}$.

\section{Terapia de rehabilitación cognitiva basada en ejercicio físico}

Palomino y col. manifestaron la eficacia de la terapia de rehabilitación basada en ejercicio físico, obteniendo resultados favorables en diversos aspectos, como función aérobica, fuerza, equilibrio, deambulación y bienestar, concluyendo que hay mejoría del cuadro clínico y exacerbación de síntomas de EP43. El ejercicio aeróbico de moderada intensidad, acompañado de ejercicios de fortaleza, beneficia al paciente con EP respecto a su capacidad motora, estado de ánimo, funciones cognitivas y patrón de sueño, por lo cual es importante abordarlo desde un estadio temprano 44

Barboza y col. llevaron a cabo un estudio con dos grupos de pacientes con síntomas motores y DC, al primero con terapia física y al segundo se le agregó entrenamiento cognitivo, demostrando mejoría en lo síntomas motores y en el DC, respectivamente, con un impacto positivo en la sintomatología, la cognición, especialmente en la memoria y en la función visoespacial, en calidad de vida y en actividades de la vida diaria de los pacientes y cuidadores ${ }^{45}$.

Conjuntamente, Olsson y col., en su investigación durante diez semanas en dos sesiones de dos horas a la semana practicando tenis de mesa, demostraron un impacto positivo en la capacidad de disfrute, salud mental y bienestar de los pacientes con DC moderado que se encontraban en la escala de Hoehn y Yahr 2 o 2,5. Fueron evaluados con los cuestionarios PDQ-8 y ED-5D-3L, por lo que se puede reconocer la relación entre el ejercicio físico y una mejor calidad de vida ${ }^{46}$.

De igual forma, Carvalho y col. determinaron la importancia de las intervenciones no farmacológicas para mejorar los síntomas motores de bradicinesia, tremor, rigidez, inestabilidad postural, además de los no motores como DC, disfunción autonómica y ansiedad por medio de rutinas de ejercicio físico, ciclismo, senderismo, caminata, artes marciales, hidroterapia y danza como terapias coadyuvantes; de esta manera tenían una calidad de vida óptima ${ }^{47}$.
En otro estudio de casos y controles efectuado por Yang y col. se compararon los efectos de la terapia con Tai Chi (ejercicio que permite tener control sobre la respiración y el cuerpo por medio de movimientos suaves y lentos) sobre los síntomas no motores de la enfermedad, como problemas para conciliar el sueño, la ansiedad, la depresión y DC. Participaron 36 sujetos con EP, colocados al azar en dos grupos: uno en entrenamiento grupal y el otro individual, durante 13 semanas, cumpliendo con tres sesiones por semana, resultando ambos en mejoría de la sintomatología no motora ${ }^{48}$.

El Ai Chi (ejercicio acuático que se centra en la respiración diafragmática y consiste en movimientos continuos y lentos para una mejor coordinación corporal) reduce el riesgo de caída (que genera inquietud en los cuidadores), beneficiando la capacidad física, las tareas cotidianas y la seguridad; incluye el uso de brazos con escaso apoyo, equilibrio y deambulación lateral en pacientes con nueve veces más posibilidades de sufrir caídas que en personas sanas del mismo grupo etario ${ }^{49}$.

Ozols y col. realizaron estudio en pacientes en estadios 1, 2 y 3 (Hoehn y Yarh), aplicando otro tipo de terapia física, las bandas elásticas, a un bajo costo, que funcionan aumentando la fuerza muscular empleando resistencia de 0,5 a 2,7 kg. Dicha herramienta permite mayor movimiento por medio de contracciones concéntricas y excéntricas. Además, refieren que existe rehabilitación de la fuerza a nivel de tren superior, movilidad, disminución de la rigidez, fortaleciendo de igual forma la independencia en ciertas actividades de la vida diaria (un hallazgo positivo tanto para el cuidador como para el paciente).

\section{Conclusiones}

Con base en la literatura consultada, los autores describen que al utilizar la terapia de rehabilitación cognitiva los pacientes con EP experimentan mejoría de los síntomas motores y no motores, aumentando no solamente su bienestar sino también el del cuidador. Al fortalecer la cognición, el paciente presenta mayor independencia para ejecutar actividades cotidianas, lo cual disminuye la carga del cuidador, su agotamiento y sentimientos de depresión, los gastos en el hogar y existe mayor empatía entre ambas partes.

Actualmente, la terapia de rehabilitación cognitiva basada en el ejercicio físico se está empleando con mayor frecuencia, debido a su bajo costo económico; en conjunto con la terapia farmacológica, el paciente mejo- 
ra su destreza y las atenciones por parte del cuidador cada vez son más espaciadas. Por lo descrito, es imperativo abordar al paciente en fase temprana para obtener una mejoría significativa y, de esa manera, enlentecer el DC y la progresión a demencia.

\section{Agradecimiento}

Agradecemos a la Dra. Cecilia Rodríguez Bellegarrigue por guiarnos en la realización de esta revisión bibliográfica, a la Universidad Dr. José Matías Delgado por brindarnos los medios y a la unión que como equipo mantuvimos para llevar a cabo este proyecto.

\section{Financiamiento}

Autoras declaran no tener fuente de financiamiento

\section{Referencias bibliográficas}

1. Montalvo J, Montalvo P, Albear L, Intriago E, Moreira D. Prevalencia de la enfermedad de Parkinson: Estudio puerta-puerta en la provincia de Manabi-Ecuador. Rev Ecuat Neurol. 2017;26(1):23-26. Disponible en: http://scielo.senescyt.gob.ec/scielo. php? script=sci arttext\&pid=S263125812017000300023\&lng=es\&nrm=iso

2. Navarta-Sánchez MV, Riverol M, Ursúa ME, Ambrosio L, Senosiain JM, Portillo MC. Intervención psicoeducativa para personas con enfermedad de Parkinson y familiares/ cuidadores: resultados preliminares de la medición basal. Rev Cient Soc Esp Enferm Neurol. 2019;49(1):16-22. DOI: 10.1016/j. sedene.2018.08.002

3. García Manzanares MC, Jiménez Navascués ML, Blanco Tobar E, Navarro Martínez M, Perosanz Calleja M. Enfermedad de Parkinson: abordaje enfermero desde atención primaria. Gerokomos. 2018;29(4):171-177. Disponible en: http://scielo.isciii.es/ scielo.php?script=sci arttext\&pid=\$1134928X2018000400171\&lng=es

4. Kouli A, Torsney KM, Kuan WL. Parkinson's Disease: Pathogenesis and Clinical Aspects. 1 ra Edición. Brisbane. Codon Publications; 2018. Capítulo 1, Parkinson's Disease: Etiology, Neuropathology, and Pathogenesis. 4-18.

5. Hurtado F, Cardenas, MAN, Cardenas FP, León LA. La Enfermedad de Parkinson: Etiología, Tratamientos y Factores Preventivos. Universitas Psychologica, 2016;15(5):1-26. DOI: 10.11144/Javeriana. upsy 15-5.epet
6. Urquizo Rodríguez El, Molina Campoverde $S$ del C, Aguirre Molina MJ, Triviño

Sánchez JS. Enfermedad de Parkinson, su asociación con los síntomas no motores. RECIAMUC. 2020;4(1):15-28. DOI: 10.26820/ reciamuc/4.(1).enero.2020.15-28

7. Lawson RA, Yarnall AJ, Johnston F, Duncan GW, Khoo TK, Collerton D, et al. Cognitive impairment in Parkinson's disease: impact on quality of life of carers. Int J Geriatr Psychiatry. 2017;32(12):1362-1370. DOI: 10.1002/gps.4623

8. Vásquez-Celaya L, Tamariz-Rodríguez A, Gutiérrez J, Marín G, Toledo M, Carrillo $P$, et al. Enfermedad de Parkinson más allá de lo motor. Revista eNeurobiología. 2019;10(23):150319. Disponible en: https:// www.uv.mx/eneurobiologia/vols/2019/23/ V\%C3\%A1squez/Nasquez-23(10)150319.pdf

9. Marín D, Carmona H, Ibarra M, Gámez M. Enfermedad de Parkinson: fisiopatología, diagnóstico y tratamiento. Rev Univ Ind Santander Salud. 2018;50(1):79-92. DOI: 10.18273/revsal.v50n1-2018008

10. Sveinbjornsdottir S. The clinical symptoms of Parkinson's disease. J. Neurochem. 2016;139(1):318-324. DOI: 10.1111/ jnc.13691

11. Muciño Bolaños MC, Meneses Rodríguez F, Sámano Osuna JA, Ayar Hernández CB, Torres Alarcón CG. Estimulación cerebral profunda en núcleo subtalámico bilateral en pacientes con enfermedad de Parkinson en el Hospital Central Militar. Rev Sanid Milit Mex. 2019;73(2):90-95. Disponible en: https://revistasanidadmilitar. org/index.php/rsm/article/view/16/17

12. Montoya-Arenas DA, Garzón Giraldo LD, Correa-López N, Carvajal-Castrillón J. Diferencias práxicas según subtipos de deterioro cognitivo en un grupo de pacientes con enfermedad de Parkinson avanzada. Med UPB. 2019;38(2):120-128. DOI: 10.18566/medupb.v38n2.a04

13. Biundo R, Weis L, Antonini A. Cognitive decline in Parkinson's disease: the complex picture. npj Parkinson's Disease. 2016;2(1):e16018. DOI: 10.1038/ npjparkd.2016.18

14. Picó Berenguer $\mathrm{M}$, Yévenes Briones $\mathrm{H}$. Trastorno del habla en enfermedad de Parkinson. Rev Cient Cienc Méd. 2019;22(1):35-42. DOI: 10.1038/ npjparkd.2016.18

15. Pigott K, Rick J, Xie S, Hurtig H, ChenPlotkin A, Duda J. Longitudinal study of normal cognition in Parkinson disease. Neurology. 2015;85(15):1276-82. DOI: 10.1212/WNL.0000000000002001

16. Muñoz Ospina BE, Orozco Vélez JL. Espectro clínico y tratamiento del trastorno cognoscitivo y demencia asociada a la enfermedad de Parkinson. 
Acta Neurol Colomb. 2019;35(3):33-46. DOI: 10.22379/24224022248

17. Mayorga-Cadavid LA, Pérez-Acosta AM. Una aproximación de la literatura científica sobre la relación entre reconocimiento de emociones, deterioro cognitivo y demencias. Cuad. neuropsicol. 2018;12(1):148-166. DOI: 10.7714/ CNPS/12.1.209

18. Bobadilla R. Desarrollo y validación de nuevas herramientas para la valoración cognitiva y el deterioro cognitivo leve en la enfermedad de Parkinson. Tesis doctoral. Barcelona. Universidad Autónoma de Barcelona; 2017.1-146p. Disponible en: https://ddd.uab.cat/pub/tesis/2017/ hdl $10803 \quad 457515 /$ rfdbm1de1.pdf

19. Smith KM, Ash S, Xie SX, Grossman M. Evaluation of Linguistic Markers of Word-Finding Difficulty and Cognition in Parkinson's Disease. J Speech Lang Hear Res.2018;13(7):1691-1699. DOI: 10.1044/2018_JSLHR-L-17-0304

20. Cancino M, Rehbein L. Factores de riesgo y precursores del Deterioro Cognitivo Leve (DCL): Una mirada sinóptica. Terapia psicológica. 2016;34(3):183-189. DOI: 10.4067/S0718-48082016000300002

21. Genç F, Yuksel B, Tokuc FEU. Caregiver Burden and Quality of Life in Early and Late Stages of Idiopathic Parkinson's Disease. Psychiatry Investig. 2019;16(4):285-291. DOI: 10.30773/pi.2019.02.20

22. Grün D, Piere V, Vaillant M, Diederich N. Contributory factors to caregiver burden in Parkinson disease. JAMDA. 2016; 17(7):626-632. DOI: 10.1016/j. jamda.2016.03.004

23. Llagostera I, López M, Sanz R, González V, Orts M. Calidad de vida y autocuidado en enfermos de Parkinson de un hospital comarcal: estudio descriptivo. Enfermería Global. 2018; (53):347-372. DOI: 10.6018/ eglobal.18.1.294561

24. Noriega C, Velasco C, Carretero I, PérezRojo G, Carretero I, Chulián A, et al. Calidad de vida, bienestar psicológico y valores en personas mayores. Revista Clínica Contemporánea. 2017;8(1):1-13. Disponible en: https:// repositorioinstitucional.ceu.es/ bitstream/10637/10656/1/Calidad CristinaNoriega et al RevClinCont 2017. pdf

25. Aponte Daza VC. Calidad de vida en la tercera edad. Ajayu. 2015;13(2):e152-182. Disponible en: $\underline{\text { http://www.scielo.org.bo/ }}$ $\mathrm{pdf} / \mathrm{rap} / \mathrm{v} 13 \mathrm{n} 2 / \mathrm{v} 13 \mathrm{n} 2 \mathrm{a} 03 . \mathrm{pdf}$

26. Palacios Sánchez E, González AV, Vicuña JA, Villamizar L. Calidad de vida en los pacientes con enfermedad de Parkinson valorados en un hospital universitario de Bogotá, Colombia. Neurol Arg.
2019;11(3):151-158. DOI: 10.1016/j. neuarg.2019.04.001

27. Cabello González C, Trandafir PC. Estudio de calidad de vida con la PDQ39 en pacientes con enfermedad de Parkinson tratados con terapias avanzadas. Rev Cient Soc Esp Enferm Neurol. 2018;48(C):9-14. DOI: 10.1016/j.sedene.2018.09.001

28. Ellingson LD, Zaman A, Stegemöller EL. Sedentary behavior and quality of life in individuals with Parkinson's disease. Neurorehabil Neural Repair. 2019;33(8):595-601. DOI: 10.1177/1545968319856893

29. Takahashi K, Kamide N, Suzuki M, Fukuda M. Quality of life in people with Parkinson's disease: the relevance of social relationships and communication. J. Phys Ther Sci.2016;28(2):541-546. DOI: 10.1589/ jpts.28.541

30. Lawson R, Yarnall A, Duncan G, Breen D, Khoo T, Williams-Gray C. Cognitive decline and quality of life in incident Parkinson's disease: The role of attention. Parkinsonism and Related Disorders 2016;27(1):47-53. DOI: 10.1016/j. parkreldis.2016.04.009

31. Limongi JCP. Quality of life in Parkinson's disease. Arq Neuropsiquiatr. 2017;75(8):493-494. DOI: 10.1590/0004$282 \times 20170114$

32. Coca SM. Calidad de vida en los cuidadores familiares de pacientes con enfermedad terminal, bajo un programa de cuidados paliativos domiciliarios. Rev. Salud Pública (Córdoba) [Internet]. 15 de diciembre de 2017 [citado 14 de julio de 2021];21(3):22-34. DOI: 10.31052/1853.1180.v21.n3.17386

33. Anaut-Bravo S, López C. El impacto del entorno residencial en la adaptación psicosocial y calidad de vida de personas cuidadores de familiares con demencia. Rev Ciencias Sociales. 2020;15(1):43-70. DOI: 10.14198/OBETS2020.15.1.02

34. Crespo-Burillo JA, Rivero Celada D, Sáenz de Cabezón A, Casado-Pellejero J, AlberdiViñas J, Alarcia-Alejos R. Influencia de la estimulación cerebral profunda en la carga de cuidadores de pacientes con enfermedad de Parkinson. Neurología. 2018;33(3):154-159. DOI: 10.1016/j. $\mathrm{nrl}$.2016.05.017

35. Espinosa D, Guzmán J, Vargas N, Ramos J. Carga de trabajo del cuidador del adulto mayor. Cina Research. 2018;2(3):28-37. Disponible en: https://journals.uninavarra. edu.co/index.php/cinaresearch/article/ view/135/69

36. Tan SB, Williams AF, Tan EK, Clark RB, Morris ME. Parkinson's Disease Caregiver Strain in Singapore. Front Neurol. 2020;11:455. DOI: 10.3389/fneur.2020.00455 
37. García-Cardoza Inocente Ismael, ZapataVázquez Rosario, Rivas-Acuña Valentina, Quevedo-Tejero Elsy del Carmen. Efectos de la terapia cognitivo-conductual en la sobrecarga del cuidador primario de adultos mayores. Horlz Sanitario. 2018;17(2):131-140. DOI: 10.19136/ hs.a17n2.2039

38. Rodríguez-Violante M, Camacho-Ordóñez A, Cervantes-Arriaga A, González-Lapatí P, Velázquez-Osuna S. Factores asociados a la calidad de vida de sujetos con enfermedad de Parkinson y a la carga en el cuidador. Neurología. 2015;30(5):e257-263. DOI: 10.1016/j.nrl.2014.01.008

39. Jiménez Carpi SV, Abreus Mora JL, GonzálezCurbelo VB, Bernal Valladares EJ, Del Sol Santiago FJ. Integración de componentes terapéuticos en la rehabilitación de pacientes con enfermedad de Parkinson. Rev Finlay. 2020;10(2): 179-190. Disponible en: http://scielo.sld.cu/scielo. php? script=sci arttext\&pid=S222124342020000200179\&lng=es

40. Lee JD, Becerra L. Tratamiento farmacológico de los síntomas motores de la enfermedad del Parkinson. Salutem Scientia Spiritus 2018; 4(1):59-61. Disponible en: https://revistas.javerianacali. edu.co/index.php/salutemscientiaspiritus/ article/view/1942/pdf

41. Mesa Valiente R, Pérez Pérez Y, Turro Mesa LN, Turro Caró E. Conducta terapéutica en ancianos con enfermedad de Parkinson. MEDISAN. 2018;22(7):614629. Disponible en: http://scielo.sld.cu/ scielo.php?script=sci arttext\&pid=S102930192018000700614\&lng=es.

42. Gurreonero Toledo CG. Programa de rehabilitación cognitiva en pacientes con Enfermedad de Parkinson. Tesis académica. Lima. Universidad Nacional Federico Villareal; 2019. 46 p.

43. Palomino S. Ejercicio físico en la rehabilitación de la enfermedad de Párkinson. Tesis doctoral. Navarra. Universidad Pública de Navarra; 2015. 76 pp. Disponible en: https://academica-e. unavarra.es/bitstream/handle/2454/18350/
TFG\%20Palomino\%20Alonso\%20Santiago. pdf? sequence $=1$ \&amp;isAllowed $=y$

44. Reynolds G, Otto M, Ellis T, Cronin A. The therapeutic potential of exercise to improve mood, cognition, and sleep in Parkinson's disease. Mov Disord. 2016;31(1):e23-38. DOI: 10.1002/ mds.26484

45. Barboza NM, Terra MB, Bueno MEB, Christofoletti G, Smaili SM. Physiotherapy versus physiotherapy plus cognitive training on cognition and quality of life in Parkinson disease. Am J Phys Med Rehabil. 2019;98:460-468. DOI: 10.1097/ PHM.0000000000001128

46. Olsson K, Franzén E, Johansson A. A pilot Study of the Feasibility and Effects of Table Tennis Training in Parkinson Disease. Arch Rehabil Research Clin Transl. 2020;2(3):100064. DOI: 10.1016/j. arrct.2020.100064

47. Carvalho Aguiar LP, Alves da Rocha P, Morris M. Therapeutic dancing for Parkinson's disease. International Journal of Gerontology. 2016;10(2):64-70. DOI: 10.1016/j.ijge.2016.02.002

48. Yang J, Wang Y, Ye S. The Effects of Group-Based versus Individual-Based Tai Chi Training on Nonmotor Symptoms in Patient with Mild to Moderate Parkinson's Disease: A Randomized Controlled Pilot Trial. Parkinson's Disease. 2017; 2017(1): 1-8. DOI: $10.1155 / 2017 / 8562867$

49. De la Llave M, Marín M, Flores L. Terapia de Ai chi para el tratamiento del equilibrio y la prevención de las caídas. Rev Investig Activ Acuát 2020;7(4):e2735. Disponible en: http://193.147.134.18/ bitstream/11000/5946/1/1718-5427-1-PB. pdf

50. Ololz M, Zúñiga C, Monterrat C, Jiménez W. Efecto de un método de entrenamiento contrarresistencia en la capacidad funcional y calidad de vida de sujetos con Parkinson idiopático. Arch Med Deporte. 2015;32(2):70-75. Disponible en: $\underline{\text { http:// }}$ archivosdemedicinadeldeporte.com/ articulos/upload/166 or01.pdf 